\title{
Research on Navigation Safety Evolution of Arctic Route Based on Entropy Theory
}

\author{
Liu Tongchao, Li Zhenfu \\ College of Transportation Management, Dalian Maritime University, Dalian 116026, China
}

\begin{abstract}
In recent years, with global warming, the development and utilization of the Arctic route has become possible. At present, the Arctic route navigation safety environment is the main factor that affects the passage of the Arctic route, so this paper mainly analyzes the development of the navigation safety environment of the Arctic route. First of all, using the fuzzy analytic hierarchy process to analyze the influence factors of the navigation safety of the Arctic route, the structure diagram of the navigation safety system of the Arctic route is divided. Secondly, according to the characteristics of navigation safety system of the Arctic route, entropy theory and fuzzy analytic hierarchy process are used to calculate the entropy values of navigation safety of Arctic routes, and the evolvement trend of navigation safety of Arctic routes is obtained. Finally, through the analysis of the trend of the Arctic route navigation security, some management countermeasures are put forward for the navigation safety of the Arctic route. The research results can provide reference for the development and utilization of the Arctic route.
\end{abstract}

\section{Introduction}

With the development of global warming and science and technology, human activities in the Arctic area are increasing day by day, and ships are sailing more frequently in the arctic. Due to the complex environment in the Arctic, the safety of ships in the Arctic region has attracted wide attention. In order to better understand the security of Arctic routes, it is necessary to analyze the evolution of the Arctic routes.

In recent years, the economic value of Arctic routes has been studied more than $[1,2,3]$. But little research for the Arctic route navigation environment, mainly Li Zhenfu respectively using the navigation environment model to explain the structure of Arctic route analysis and comprehensive safety evaluation method of Arctic route navigation environment by fuzzy comprehensive evaluation [4,5]. The existing assessment of the Arctic sea route safety environment mostly focuses on the evaluation of the navigation environment of the Arctic route without taking into consideration the factors of the Arctic route and the participation of management factors. The data show that $80 \%$ of the accidents are caused by human factors, $80 \%$ of which are caused by improper management. Therefore, although the geographical environment of the Arctic route is very important for the assessment of the security environment of the Arctic routes, human factors and management factors are also indispensable.

The navigation safety of the Arctic route is a systematic project, and the navigation safety of the Arctic route should be analyzed from the point of view of the system. Therefore this paper on Arctic route navigation safety evolution analysis method and safety entropy theory combining fuzzy analytic hierarchy process, and the use of safety system management method for the demonstration of the evolution trend, put forward the Arctic route navigation safety management countermeasures.

\section{Theoretical}

\subsection{FAHP}

AHP is widely used in multi attribute decision making method, but does not take into account the ambiguity of the people when they make decisions accordingly, fuzzy set theory and AHP combined with Fuzzy AHP method is proposed to fully considering the fuzziness of expert decision-making, and effectively reduce the AHP the inconsistency. Fuzzy analytic hierarchy process can analyze the system, divide the subsystems of different levels, and calculate the weights of different attributes [6].

\subsection{Safety entropy theory}

Entropy was put forward by T.Clausius in 1854. It is mainly used to describe the state of a system, and the order of the system state is represented by the entropy value. Originally, entropy was mainly applied to statistical physics and thermodynamics, and later expanded to biology, information theory and astrophysics, and then extended to various fields of natural science and some fields of social science. Entropy theory belongs to the initial stage in the application of security field. In this 
paper, we will expand the application of the safety entropy to analyze the evolution mechanism of the navigation safety system of the Arctic route.

\subsection{Safety system management}

Security system management is a comprehensive approach to ensure the safe operation of the system, mainly by using the laws and principles of safety science, system science and management science [7]. The main method of analysis is to analyze the state of the security system first, secondly, analyze the hidden security problems in the security system, and finally put forward some management countermeasures to the security system by using management methods.

\section{Computing method}

Firstly, the fuzzy analytic hierarchy process is used to analyze the system. Fuzzy AHP is used to analyze the hierarchical structure of the problem, and the complex problem is decomposed into several relatively simple sub structures. And through the fuzzy comparison of the 22 elements, and then obtain the weight of each element, and then get the relative important order. Fuzzy analytic hierarchy process (AHP) is a widely used method for partitioning the weights between different attributes.

The definition of [8] set matrix is an interval judgment matrix:

$$
M=\left[\begin{array}{cccc}
1 & {\left[l_{12}, u_{12}\right]} & \ldots & {\left[l_{1 n}, u_{1 n}\right]} \\
{\left[l_{21}, u_{22}\right]} & 1 & \ldots & {\left[l_{2 n}, u_{2 n}\right]} \\
\vdots & \vdots & \ddots & \vdots \\
{\left[l_{n 1}, u_{n 1}\right]} & {\left[l_{n 2}, u_{n 2}\right]} & \ldots & 1
\end{array}\right]
$$

Where the upper and lower bounds of the interval values are represented respectively. If satisfied,

The interval judgment matrix is the interval reciprocal judgment matrix. The calculation of matrix, [9] Islam's method, this method can not only solve the consistency of interval reciprocal judgment matrix, but also can not satisfy the consistency for interval complementary judgment matrix is obtained by the weight vector is calculated as follows:

$$
\begin{array}{ll}
\min & \sum_{i=1}^{n-1} \sum_{j=i+1}^{n}\left(p_{i j}+q_{i j}\right) \\
\text { s.t. } & w_{i}-l_{i j} w_{j}+q_{i j} \geq 0, i=1,2, \ldots, n-1 ; j=i+1, i+2, \ldots, n \\
& w_{i}-u_{i j} w_{j}-p_{i j} \leq 0, i=1,2, \ldots, n-1 ; j=i+1, i+2, \ldots, n \\
& \sum_{i=1}^{n} w_{i}=1, j=1,2, \ldots, n \\
& w_{i}, p_{i j}, q_{i j} \geq 0, i, j=1,2, \ldots, n
\end{array}
$$

It represents the offset distance that deviates from the lower bound of the interval; represents the deviation distance that deviates from the upper bound of the interval; it is the weight of different attributes.

Secondly, the utility function is used to calculate each attribute value, and different kinds of data are calculated. The concrete formula is as follows:

$$
\begin{gathered}
u_{i}=1+\min \left(x_{i}\right) / \max \left(x_{i}\right)-x_{i} / \max \left(x_{i}\right) \\
u_{i}=x_{i} / \max \left(x_{i}\right)
\end{gathered}
$$

Thirdly, the entropy theory is used to calculate the entropy of each attribute of the security system.

$$
S\left(x_{i}\right)=k \log 1 / P\left(x_{i}\right)=-k \log P\left(x_{i}\right)(i=1,2, \cdots n)
$$

The article is a factor of safety; safety is the safety factor of the entropy is a factor of safety; safety; undetermined coefficient is, is an undetermined coefficient in order to facilitate and other combination of theory, which can be assigned to 1 . The total entropy of safety can be weighted by the sum of the entropy of different attributes, and the concrete formula is as follows:

$$
S=-\sum w_{i} \log P\left(x_{i}\right)(i=1,2, \cdots n)
$$

Among them is the weight of the first attribute, which can be obtained by formula (1).
Only when the total entropy of the security system $S=0$, the security system in a most secure state, the system can be safe and orderly development of stability. The probability of occurrence of a safety accident is at its lowest level. Since the minimum value $S$ is 0 , the greater the value in any case $S \geq 0$, the more insecure the system state is $S$. The following analysis of the safety system changes according to different situations.

Suppose scenario 1 , when $S_{t}=a,(a \geq 0), S_{t+1}>a$, that is $d S / d t>0$, represents the security system in the direction of instability, the system is increasingly insecure.

When $d^{2} S / d t^{2}>0$, the state of the safety system was shown to move towards a unstable state at a faster rate.

When $d^{2} S / d t^{2}=0$, the state of the safety system was shown to move to a steady state at a steady rate. In this case, there are not many in the real world

When $d^{2} S / d t^{2}<0$, the state of the safety system was shown to move towards a state of instability at a slower rate. 
Suppose scenario 2, when $S_{t}=a,(a \geq 0), S_{t+1}<a$,the value $S$ is reduced. $d S / d t<0$ represents the development of a security system toward a stable approach, and the system is becoming less secure.

When $d^{2} S / d t^{2}>0$, the state of the safety system was shown to move towards a unstable state at a faster rate.

When $d^{2} S / d t^{2}=0$, the state of the safety system was shown to move to a steady state at a steady rate.

When $d^{2} S / d t^{2}<0$, the state of the safety system was shown to move towards a state of instability at a slower rate.

To sum up, for the analysis of system security evolution, the steps are as follows:

Step 1 calculate the attribute weight of the security system according to formula (1)

Step 2 normalize the attribute values of the security system according to the formula (2)

step 3, according to formula (3) and (4), each attribute value of the security system is calculated by entropy

Step 4 calculate the total entropy of the safety system according to formula (5)

Step 5, according to different system security change scenarios, make different security management countermeasures.

\section{Calculation and analysis of navigation}
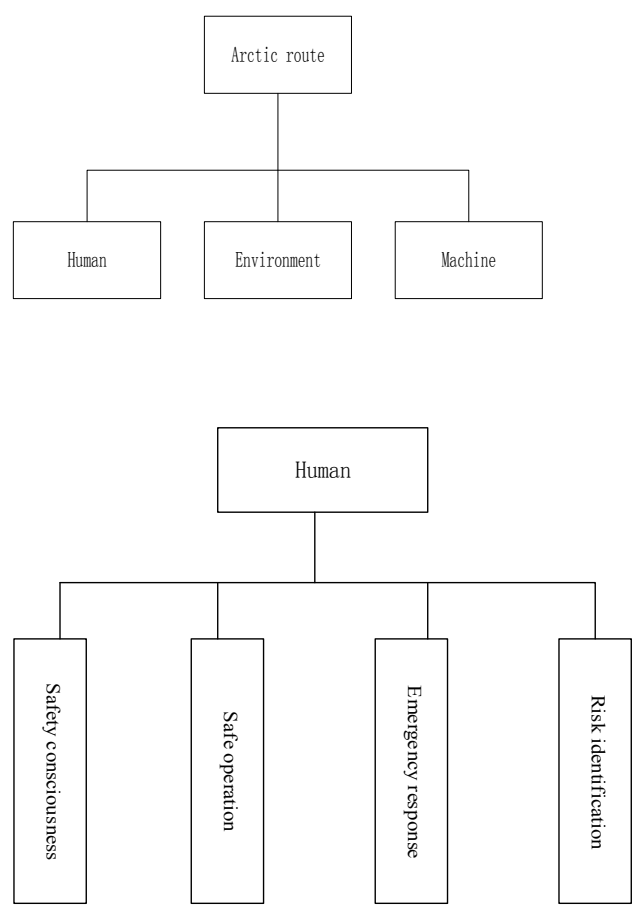

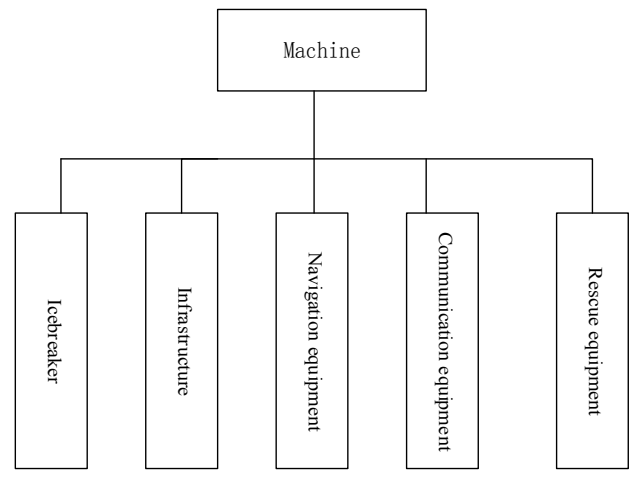

\section{safety system evolution of Arctic route}

\section{1 evolution calculation of navigation safety system of Arctic route}

The security environment of the Arctic route plays an important role in the navigation and exploitation of the Arctic route. Therefore, it is very necessary to evaluate the security environment of the Arctic route. There are many factors affecting the safe navigation of the Arctic route. This paper uses fuzzy analytic hierarchy process (AHP) to systematically analyze the security status of the Arctic routes from three aspects, such as human, machine and environment. From the analysis of human factors, the main factors included safety awareness, safety operation, emergency response, risk identification and improper behavior. From the factor analysis of aircraft, the main factors included ice breaker, port infrastructure, navigation instruments, communication equipment and rescue equipment. From the analysis of environmental factors, the factors included ice, visibility, wind, channel width and water depth, channel obstacles, tides and currents, polar front and cyclones. The detailed structure is shown in figure 1 . The Arctic route number of ship navigation data mainly from the northern sea route official information (http://www.arctic-lio.com/nsr_transits), ice area data mainly comes from the National Snow and Ice Data Center (https://nsidc.org/data).

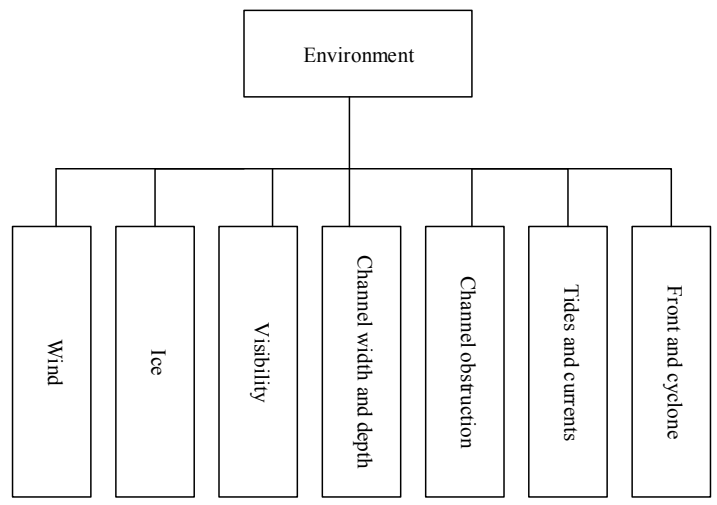

Fig.1. Arctic route safety structure 
Table 1. Fixed value of human factors

\begin{tabular}{|l|c|c|c|c|}
\hline & safety consciousness & Safe operation & Emergency response & Risk identification \\
\hline Utility value & 0.84 & 0.86 & 0.76 & 0.88 \\
\hline
\end{tabular}

Table 2. Fixed value of machine factors

\begin{tabular}{|c|c|c|c|c|c|}
\hline & Icebreaker & Infrastructure & Navigation equipment & Communication equipment & $\begin{array}{c}\text { Rescue } \\
\text { equipment }\end{array}$ \\
\hline Utility value & 0.77 & 0.65 & 0.75 & 0.68 & 0.70 \\
\hline
\end{tabular}

Table 3. Fixed value of environmental factors

\begin{tabular}{|l|c|c|c|c|c|c|}
\hline & wind & visibility & $\begin{array}{c}\text { Channel width and } \\
\text { depth }\end{array}$ & $\begin{array}{c}\text { Channel } \\
\text { obstruction }\end{array}$ & $\begin{array}{c}\text { Tides and } \\
\text { currents }\end{array}$ & $\begin{array}{c}\text { Front and } \\
\text { cyclone }\end{array}$ \\
\hline Utility value & 0.68 & 0.64 & 0.80 & 0.76 & 0.74 & 0.70 \\
\hline
\end{tabular}

Table 4. Average area of ice per year

\begin{tabular}{|l|l|}
\hline Year & Ice area \\
\hline 2003 & 11.640 \\
\hline 2004 & 11.443 \\
\hline 2005 & 11.186 \\
\hline 2006 & 11.041 \\
\hline 2007 & 10.753 \\
\hline 2008 & 11.262 \\
\hline 2009 & 11.233 \\
\hline 2010 & 10.968 \\
\hline 2011 & 10.732 \\
\hline 2012 & 10.695 \\
\hline 2013 & 11.150 \\
\hline 2014 & 11.047 \\
\hline 2015 & 10.823 \\
\hline 2016 & 10.384 \\
\hline
\end{tabular}

Table 5. Number of shipping traffic per year

\begin{tabular}{|c|c|}
\hline Year & Ship number \\
\hline 2009 & 2 \\
\hline 2010 & 4 \\
\hline 2011 & 41 \\
\hline 2012 & 46 \\
\hline 2013 & 71 \\
\hline 2014 & 31 \\
\hline 2015 & 18 \\
\hline
\end{tabular}

Table 6. Ice area standardization

\begin{tabular}{|c|c|}
\hline Year & Ice area \\
\hline 2003 & 0.30 \\
\hline 2004 & 0.31 \\
\hline 2005 & 0.33 \\
\hline 2006 & 0.34 \\
\hline 2007 & 0.37 \\
\hline 2008 & 0.33 \\
\hline 2009 & 0.33 \\
\hline 2010 & 0.35 \\
\hline 2011 & 0.37 \\
\hline 2012 & 0.37 \\
\hline 2013 & 0.34 \\
\hline 2014 & 0.34 \\
\hline 2015 & 0.36 \\
\hline 2016 & 0.39 \\
\hline
\end{tabular}

Table 7. Ship quantity standardization

\begin{tabular}{|c|c|}
\hline Year & Ship quantity \\
\hline 2009 & 1 \\
\hline 2010 & 0.99 \\
\hline 2011 & 0.95 \\
\hline 2012 & 0.95 \\
\hline 2013 & 0.91 \\
\hline 2014 & 0.96 \\
\hline 2015 & 0.98 \\
\hline
\end{tabular}


Table 8. The total entropy of safety system in different years

\begin{tabular}{|c|c|}
\hline Year & Safety entorpy \\
\hline 2002 & 0.158 \\
\hline 2003 & 0.158 \\
\hline 2004 & 0.158 \\
\hline 2005 & 0.153 \\
\hline 2006 & 0.152 \\
\hline 2007 & 0.150 \\
\hline 2008 & 0.154 \\
\hline 2009 & 0.153 \\
\hline 2010 & 0.151 \\
\hline 2011 & 0.149 \\
\hline 2012 & 0.150 \\
\hline 2013 & 0.154 \\
\hline 2014 & 0.154 \\
\hline 2015 & 0.151 \\
\hline 2016 & 0.147 \\
\hline & \\
\hline
\end{tabular}

\section{2 evolution analysis of navigation safety system of Arctic route}

You can see from Figure 2 in the Arctic route navigation safety system in general is gradually to the safe state, but can be seen from the figure figure the evolution trend of the Arctic route navigation safety system has two fluctuations, data can be seen from the first wave is affected by the ice changes the whole Arctic ice area is decreasing, but the first time in 2008 and 2009 when the fluctuation of ice area more than a few years ago the ice area is large, so the Arctic route navigation safety system to the entropy fluctuations, but after the ice area declined, the Arctic route navigation safety system entropy correspondingly decline. For the second time in terms of fluctuation is mainly affected by the number of ship of the Arctic route, the number of ship navigation from 2009 to 2013 the Arctic route has increased year by year, so the Arctic route navigation safety system to the entropy fluctuations, but because the number of ship navigation and ice area decreased, while the Arctic route navigation safety system and entropy a downward trend. At present, with the melting of the Arctic ice layer, the navigation environment of the Arctic route will be more and more safe. However, with the increase of navigation

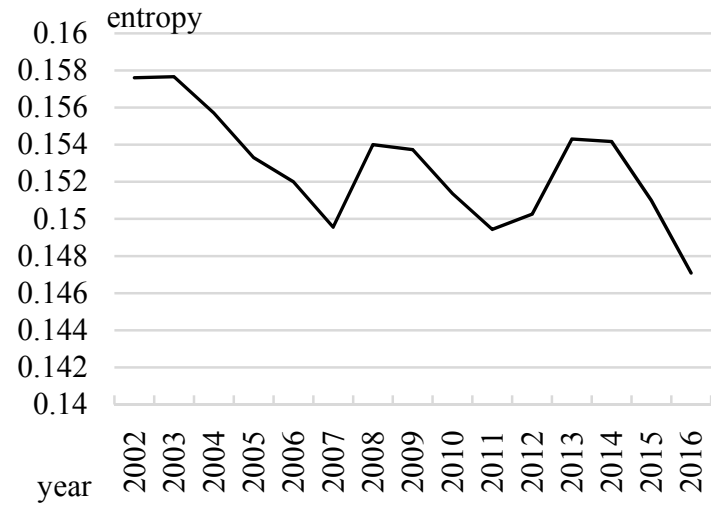

Fig.2. The evolution of the Arctic route navigation safety system

ships, the navigation safety system of the Arctic route will inevitably be affected.

The analysis in Figure 2 except fluctuations in the region outside of the Arctic route navigation safety system entropy change, from Figure 2 can be found, decreased from 2002 to 2007, the Arctic route navigation safety system entropy, and from the figure can be found in the Arctic route navigation safety system based on the linear entropy decrease, namely, that the Arctic route navigation safety system the Arctic ice area of straight down. From 2014 to 2016 declined, the Arctic route navigation safety system entropy, and from the figure can be found in the Arctic route navigation safety system entropy basic straight down, that is, during this period the Arctic route navigation safety system from the Arctic ice area is reduced and the ship reduced the number of double effect, the Arctic route navigation safety system entropy appears sharp drop.

Overall, the Arctic route navigation safety system state change rate is not large, the main reason is the ice changes in the Arctic route navigation safety system occupies the proportion is 0.09 , impact on the Arctic route navigation safety system is limited. There are many factors in the navigation safety system of the Arctic route, 
and various factors influence the change of the security system of the Arctic route synthetically. You can see from Figure 2 that the Arctic route navigation safety system is in the transition phase to a safe condition, according to the analysis of scenarios, for the Arctic route navigation safety system, this should continue to implement the management of Arctic route area, and according to some problems on the management of repair.

\subsection{Management Countermeasures of navigation safety system in Arctic route}

Although with the melting of Arctic ice, Arctic route navigation safety system is in the transition phase to a safe condition, but the Arctic route navigation safety system there are still many problems, such as the Arctic route along the port infrastructure is seriously insufficient, the sovereignty issue, such as lack of communication equipment. In view of these problems, the following suggestions are put forward.

First, we should strengthen infrastructure construction along the Arctic route and improve the transportation system of the Arctic route. At present, the port infrastructure along the Arctic route can not meet the safety of commercial vessel docking, and the infrastructure of the ports along the Arctic route should be strengthened. Second, strengthen the legal construction of the Arctic route and improve the legal system of the Arctic route. At present, there are very many laws about the Arctic routes, but these laws vary in quality and even contradict each other. Third, we should strengthen the monitoring of natural environment in the Arctic region and improve the accuracy of arctic weather prediction. For ship navigation safety in the Arctic, the biggest security threat is from the natural environment of the Arctic, the Arctic climate, be the most changeful, with great uncertainty, strengthen environmental monitoring can effectively reduce the number of ships sailing in the extreme weather or the ship in advance to take preventive measures to cope with the extreme weather, and safeguard people's safety and property safety.

\section{5 conclusion}

Based on the entropy theory, the application of existing entropy theory in the field of security development, put forward the analysis method of security system evolution, and using this method the evolution trend of Arctic route navigation safety system are analyzed, the results found that the Arctic route navigation safety system is in the transition phase to a safe condition. However, there are still some hidden dangers in the navigation safety system of the Arctic route. Therefore, this paper puts forward some management countermeasures for the navigation safety system of the Arctic route. In addition, the evolution of the navigation safety of the Arctic route in space is worthy of further attention.

\section{References}

1. Wang N, Yan B, Wu N, et al. Transport. Res. Part A Pol. Pract., 2016.

2. Lasserre F. Transport. Res. Part A Pol. Pract., 2016.

3. Lasserre F. Transport. Res. Part A Pol. Pract. 66(1):144-161. 2014.

4. LI Z F, LI Y, YU S Q. World Reg Stu, 22(2):11-17. 2013.

5. LI Z F, YAN L, XU M Q, et al. Comput Eng Appli,, 49(1):249-253. 2013

6. Tavana M, Zareinejad M, Caprio D D, et al. Appl Soft Comput, 40:544-557. 2016.

7. ZHANG S, SHI X Z, W C.Chin.Saf.Sci.J. 06:9-16. 2010.

8. Salo A A, Hämäläinen R P.. Eur.J.Oper.Res.,82(3): 458-475.1995.

9. Islam R, Biswal M, Alam S. Eur.J.Oper.Res, 97(1): 53-62. 1997. 